\title{
New And/Or Interesting at the MSA/MAS '94 Conference
}

\author{
For the interest for readers who were not able to attend the recent MSAMMAS '94 Conference in New Orleans, we attempt as follows to \\ summarize what was "new and/or interesting".
}

$\checkmark$ AMRAY, INC. displayed its new model 1920 ECO-SEM. Fundamentally and at the push of a button, will convert to a low vacuum mode for the imaging research including their unique Oscillating Tissue Slicer and Laboratory Miof non-conductive samples in their natural state. Tel.: (800)225- crowave Oven. Registration for their newest 300 page plus catalog due out this 1462/(617)275-1400, Fax: (617)275-0740. Circle Reader Inquiry \#3

$\checkmark$ Burleigh Instruments, Inc. exhibited the line of Personal Scanning Probe Microscopy (PSPM) products. This new generation of microscopes includes an AFM, STM, and UHV/STM. They are all extremely affordable, high performance microscopes designed for routine imaging of fine surface topography with precise 3-D quantification. Also shown was the STM product designed for the undergraduate classroom and research laboratory. Tel.: (716)924-9355, Fax: (716)924-9072. Circle Reader Inquiry \#18

$\checkmark$ Carl Zeiss, Electron Optics Div.: The new Carl Zeiss DSM 982 - Gemini FEG/SEM was shown for the first time in the USA. The response to this distinctive instrument ... with 5 nanometers resolution at only 500 volts ... indicates it has become the new technical leader in FEG Scanning Electron Microscopy. Tel.: (800)356-1090, Fax: (914)681-7442. Circle Reader Inquiry \#2

$\checkmark$ Dapple Systems: The Micro.EDS updated graphical presentation now includes a unique 3-D display for comparison of 6 spectra overlayed or stacked. Quantitative results are easily checked by displaying the fitted spectra and resulting residual against the original. Tel.: (408)733-3282, Fax: (408)736-2350. Circle Reader Inquiry \#8

$\checkmark$ Diatome, USA had on display their complete collection of Diamond Knives for electron and light microscopy at room and cryo temperatures. All of the knives were in a multitude of angles offering the microtomy community a knife for every application. Tel.: (800)523-5874/(215)646-1478, Fax: (215)646-8931. Circle Reader Inquiry \#19

$\checkmark$ Digital Instruments exhibited its complete line of NanoScope ${ }^{\circ}$ Scanning Probe Microscopes (SPMs), including the MultiMode ${ }^{\top M}$ Atomic Force Microscope (AFM). Also featured were the new BioScope ${ }^{\text {TM }}$ AFM and Dimension ${ }^{T M}$ 3000 SPM. The Bioscope system is the first probe microscope specifically designed for biotechnology and life sciences. The Dimension AFM offers a complete range of AFM techniques - including magnetic force, lateral force, electrochemistry, and others - for small or large samples and at a mid-range price. Tel.: (805)899-3380, Fax: (805)899-3392. Circle Reader Inquiry \#7

$\checkmark$ Delaware Diamond Knives, Inc. (DDK) manufactures the entire range of diamond knives. Choose your knife and boat from ultrathin or thick ... high to low angle ... ambient or cryo ... wet or dry. All styles are guaranteed for unlimited resharpenings and for your application. The excellent results from the PS1000 for in-situ cryofixation were shown. Affordability and versatility make the PS1000 perfect whether you are experienced or new to cryotechniques. Tel:: (800)222-5143/(302)999-7476, Fax: (302)999-8320. Circle Reader Inquiry \#12

fall is being accepted. Please call to register. Tel.: (215)646-1566, Fax: (215)646-8931. Circle Reader Inquiry \#22

$\checkmark$ ETP- USA showed the new Series 4 Robinson Detector operating with the new microprocessor joy stick controller as well as computer driven WINDOWS. Also on display was the new SERIES 4 Infrared CHAMBER VIEW SYSTEM with non-glare illumination and large depth of field. Tel.: (510)449-8534 or (800)8 ETP USA. Fax: (510)449-8996. Circle Reader Inquiry \#13

$\checkmark$ FEl Company introduced Focused Ion Beam technology by demonstrating their new FIB200 workstation. FEl's $20 \mathrm{~nm}$ resolution ion beam is used to micromachine materials ranging from animal tissue to semiconductors, precisely exposing buried submicron features for TEM and SEM analysis. FEl's ion beam imaging capability delivered stunningly clear high contrast images at magnifications similar to electron microscopes. Tel.: (503)640-7500, Fax: (503)640-7509. Circle Reader Inquiry \#6

$\checkmark$ Fisons Instruments Mfg., Inc.: Kevex introduced the new SuperQuantek ambient pressure detector window for EDS detectors which offers the best light element transmission of any window currently on the market. This window is available for LN or SuperDry detectors, new or retrofit. Tel.: (805)295-0019, Fax: (805)295-8714. Circle Reader Inquiry \#14

$\checkmark$ 4pi Analysis, Inc. introduced the Spectral Engine ${ }^{\text {TM }}$ II, providing improved $x$-ray (EDS) and/or digital image acquisition directly into a Macintosh. New x-ray image mapping capabilities were announced also. Can easily upgrade older existing systems or be installed on new systems as well. Tel.: (919)489-1757, Fax: (919)489-1487. Circle Reader Inquiry \#23

$\checkmark$ Harris Diamond Corporation is proud to introduce the Drukker diamond untramictotome knife manufactured by Drukker International B.V., The Netherlands. Advantages include superb wetting behavior and guaranteed compression-free sections of soft biological tissue. An immediate exchange program is offered, whereby used knives are accepted for new Drukker knives. New knives are guaranteed to ship within 2 weeks. Tel.: (201)770-1420, Fax: (201)770-1549. Circle Reader Inquiry \#24

$\checkmark$ IMAGRAPH Corporation demonstrated the IMASCAN family of high performance, cost-effective add-in cards for integrated VGA display and video capture applications. IMASCAN's programmable input is compatible with a wide variety of microscopes. Image Analysis bundles are available with Media Cybernetics Image-Pro Plus and a driver is also available for Optimas. IMASCAN supports $24 / 16 / 8$ bit display at resolutions up to $1280 \times 1024$ and is available in $\mathrm{VL}$ or $\mathrm{PCl}$ bus versions. Tel:: (508)256-4624, Fax: (508)250-9155. Circle Reader Inquiry \#25

$\checkmark$ EDAX International: Major new products for X-ray microanalysis introduced included the PowerMX system based on the Apple PowerPC and the new $L N$ free detector, the CryoX. New software packages for automated analysis, including GSR, were also introduced. Tel.: (201)529-6231, Fax: (201)529-3156. Circle Reader Inquiry \#20

$\checkmark$ E. FJELD Co., Inc. displayed a pair of 6 -axis high resolution specimen stages. The sixth axis is an additional tilt axis perpendicular to the main tilt of the stage to optimize geometries for EDS detectors, stereo imaging and crystallography studies. In addition, a series of metallurgical mounts, a CCD infrared camera and Faraday assembly were displayed. Tel.: (508)667-1416, Fax: (508)667-9059. Circle Reader Inquiry \#21

$\checkmark$ JEOL USA premiered its new UNIX based SEM, the X-VISION. This new instrument, which is a natural extension of JEOL's successful VISION product, is the worlds' first UNIX based SEM. Features include true 32 bit speed, multitasking, high performance digital imaging and processing and many more features that are made possible by the power of the UNIX operating system. Tel:: ((508)535-5900, Fax: (508)536-2205. Circle Reader Inquiry \#5

$\checkmark$ Leica, Inc. featured sime exciting new products, such as the $\$ 400$ series scanning electron microscopes, fully integrated (Windows based) with Fisons and Oxford EDS systems, and SEM image analysis software. Also on display were Leica's new ultramicrotome and freeze drying systems. Leica demonstrated a laser confocal scanner on an inverted microscope. Tel.: (800)248-0123, Fax: (708)405-0030. Circle Reader Inquiry \#4 
$\checkmark$ Material Analytical Services (MAS) announces the introduction of new instrumentation and engineering services for microscopists. Instrumentation

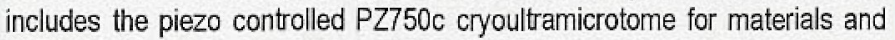
biomedical applications and the VC1000 Versicoater for advanced materials applications. Also available are high aspect ratio milled single crystal silicon SPM tips for resolving deep trenches in tapping or non-tapping modes. Tel.: (800)421-8451, Fax: (404)368-8256. Circle Reader Inquiry \#10

$\checkmark$ Noesis Vision, Inc.: Visilog is an image processing software package running under Windows 3.1, NT and X-windows. Visilog provides an extensive selection of image processing algortihms. A powerful $\mathrm{C}$ interpreter allows for automatic recording and $\mathrm{C}$ programming. Extension modules: advanced morphology, segmentation, color, fiber analysis, ASTM grain sizing and Correlation Hough Transforms. Tel.: (514)345-1400, Fax: (514)345-1575. Circle Reader Inquiry \#27

$\checkmark$ Oxford Instruments introduced Cameo, a radically different approach to microanalysis, which gives a complete picture of both the topography and chemical composition of a sample simultaneously. Also seen for the first time was Theta, a product which dramatically simplifies combined EDMD analysis by combining the control and display of the EDS and WDS systems in the same user interface. Tel.: (508)369-9933, Fax: (508)369-8287. Circle Reader Inquiry \#28

$\checkmark$ RJ Lee Group, Inc.: The PERSONAL SEM ${ }^{(3)}$ is a low-cost, all digital scanning electron microscope that is easy to operate and maintain. Intimidating controls of the traditional SEM have been replaced by graphical "point and click" operation and disposable filaments and column liner tubes can be easily replaced by the operator. Tel.: (412)744-0100, Fax: (412)744-0506. Circle Reader Inquiry \#29

$\checkmark$ Small World introduced the "Electron Flight Simulator", the first Monte Carlo modeling software for Windows. Now you can model $x$-ray generation from bulk, thin film or film on a substrate samples at any tilt, any $k \mathrm{~V}$ and any sample chemistry. Normally $\$ 495$ but take $30 \%$ off with the MSA discount, now only $\$ 350$ if ordered prior to Sept. 30 ' 94. Tel./Fax: (415)345-8013. Circle Reader Inquiry \#30

$\checkmark$ Ted Pella, Inc.: A new Microwave Oven system was introduced with water load monitoring in a unique temperature probe development that can be connected to a computer. These offer improved reproducibility in specimen preparation. A Microwave Oven/EM Bulletin Board was announced for access in September, access number is (916)243-9456. Tel.: (916)243-2200, Fax: (916)243-3761. Circle Reader Inquiry \#31

$\checkmark$ Topometrix: The Observer ${ }^{T M}$ SPM for SEM was displayed at both the Topometrix and Hitachi Scientific Instruments booths. Observer is the industry's only SPM designed specifically to be used inside a SEM chamber. The instruments displayed were configured for use with Hitachi's models S-4100, S-4200 and S-4500-II SEMs. The company announced that they had entered negotiations with several firms for system integration of Observer with other SEM models.. Tel.: (408)982-9700, Fax: (408)982-9751. Circle Reader Inquiry \#1

$\checkmark$ Virtual Laboratories, manufacturer of the world's most comprehensive program for diffraction simulation and analysis, demonstrated Desktop Microscopist. Premiering was the native mode version for the Macintosh Power $\mathrm{PC}$, along with a preview of the dislocation module to be released later this year. Also announced was the availability of a network version. Tel.: (505)828-1640, Fax: (505)822-9759. Circle Reader Inquiry \#9

$\checkmark$ Vital Image Technology, Inc. introduced VitalScan, a $\$ 9,995 \mathrm{PC}$ based imaging system, to modernize analog SEMs with digital imaging technology. Secondary and backscatter images can be collected as high as $4 K x$ $4 \mathrm{~K}$ with 12 -bit digitization. Options include faxt -xray mapping, image analysis software and mass storage devices. V.I.T. also photographic-quality printers and other imaging products including CCD cameras and frame grabber cards. Tel: (800)860-IMAGE, Fax: (216)786-7799. West Coast, Tel: (805)2975531, Fax: (805)297-3814. Circle Reader Inquiry \#11

\section{EM PRODUCT MANAGER}

\section{AND \\ SEM DEMONSTRATOR}

Carl Zeiss, Inc., Electron Optics Division, is seeking an individual to fill the key position of EM Product Manager and SEM Demonstrator at the Thornwood, New York facility. Minimum requirements include:

Masters Degree (Ph.D. preferred).

Well versed in TEM and SEM procedures.

Willing to travel.

Capable of supervising the activities of the

TEM senior applications specialists.

Enjoy working with people.

Well groomed and articulate.

Send resume, in complete confidence, to:

Mr. Paul Henry, General Manager

Carl Zeiss, Inc., Electron Optics Division

One Zeiss Drive

Thornwood, N.Y. 10594

Tel.: (914)681-7741 - Fax: (914)681-7443

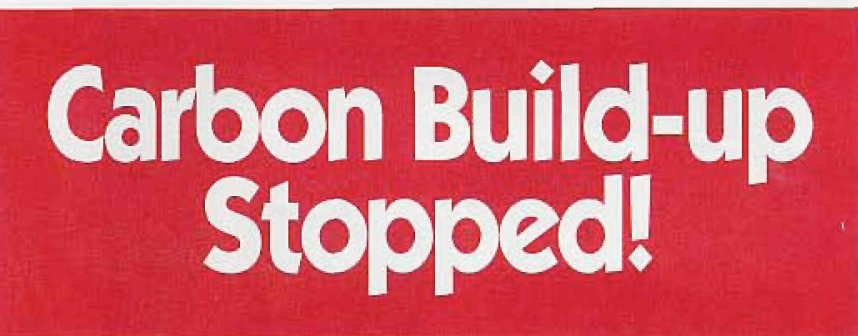

Applications Lab Secret Revealed:
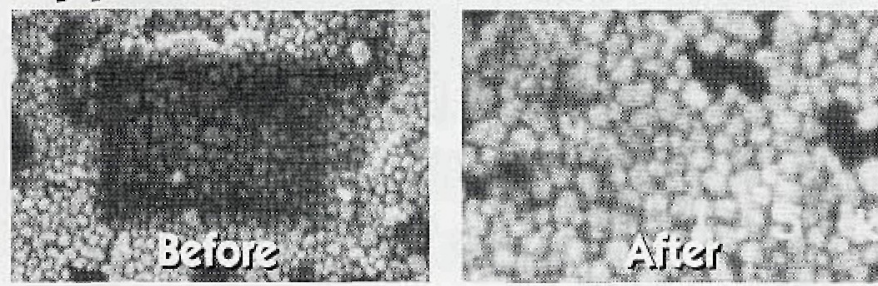

SEM manufacturers won't admit it, but most SEMs are subject to contamination build-up-even dry pumped systems. To stop hydrocarbon condensation, major applications labs and SEM users rely on the XEI Scientific SEM-CLEAN ${ }^{\top M}$ system.

Result: Outstanding pictures at low $\mathrm{kV}$ and high resolution and no oil on EDS X-ray detector windows. The Nitrogen purge of the inexpensive SEM-CLEAN system cleans your electron microscope while you're away

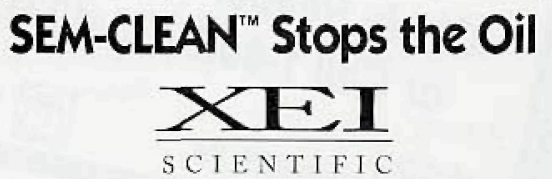

3124 Wessex Way, Redwood City, CA 94061 - 415-369-0133 - Fax 415-363-1659 